\title{
Real-World Comparisons of Low-Dose NOACs versus Standard-Dose NOACs or Warfarin on Efficacy and Safety in Patients with AF: A Meta-Analysis
}

\author{
Ze Li $\mathbb{D}^{1},{ }^{1}$ Xiaozhen Wang $\left(\mathbb{D},{ }^{2}\right.$ Dandan $\mathrm{Li}\left(\mathbb{D},{ }^{1}\right.$ and Aiping Wen $\mathbb{D}^{1}$ \\ ${ }^{1}$ Department of Pharmacy, Beijing Friendship Hospital, Capital Medical University, Beijing, China \\ ${ }^{2}$ Central Laboratory, Xuanwu Hospital, Capital Medical University, Beijing, China \\ Correspondence should be addressed to Aiping Wen; wenaiping@ccmu.edu.cn
}

Received 29 November 2021; Accepted 9 February 2022; Published 7 March 2022

Academic Editor: Domenico Della Rocca

Copyright (c) $2022 \mathrm{Ze} \mathrm{Li} \mathrm{et} \mathrm{al.} \mathrm{This} \mathrm{is} \mathrm{an} \mathrm{open} \mathrm{access} \mathrm{article} \mathrm{distributed} \mathrm{under} \mathrm{the} \mathrm{Creative} \mathrm{Commons} \mathrm{Attribution} \mathrm{License,} \mathrm{which}$ permits unrestricted use, distribution, and reproduction in any medium, provided the original work is properly cited.

Objective. We aimed to further investigate the efficacy and safety of low-dose NOACs by performing a meta-analysis of cohort studies. Background. Meta-analyses of randomized controlled trials (RCTs) have demonstrated that low-dose non-vitamin K antagonist oral anticoagulants (NOACs) showed inferior efficacy compared with standard-dose NOACs, although they are still frequently prescribed for patients with atrial fibrillation (AF) in the clinical practice. Methods. Cochrane Central Register of Controlled Trials (CENTRAL), Embase, and MEDLINE were systematically searched from the inception to September 9, 2021, for cohort studies that compared the efficacy and/or safety of low-dose NOACs in patients with AF. The primary outcomes were ischemic stroke and major bleeding, and the secondary outcomes were mortality, intracranial hemorrhage (ICH), and gastrointestinal hemorrhage (GH). Hazard ratios (HRs) and 95\% confidence intervals (CIs) were estimated with the random-effect model. Results. Twenty-five publications involving 487856 patients with AF were included. Compared with standard-dose NOACs, low-dose NOACs had comparable risks of ischemic stroke (HR=1.03, 95\% CI 0.96 to 1.11), major bleeding $(\mathrm{HR}=1.12$, 95\% CI 0.97 to 1.28$)$, ICH ( $\mathrm{HR}=1.09,95 \%$ CI 0.88 to 1.36$)$, and $\mathrm{GH}(\mathrm{HR}=1.11,95 \%$ CI 0.92 to 1.33$)$, except for a higher risk of mortality ( $\mathrm{HR}=1.41,95 \%$ CI 1.21 to 1.65$)$. Compared with warfarin, low-dose NOACs were associated with lower risks of ischemic stroke ( $\mathrm{HR}=0.72,95 \% \mathrm{CI} .67$ to 0.78$)$, mortality ( $\mathrm{HR}=0.67,95 \% \mathrm{CI} 0.59$ to 0.77$)$, major bleeding $(\mathrm{HR}=0.64,95 \% \mathrm{CI}$ 0.53 to 0.79$)$, $\mathrm{ICH}(\mathrm{HR}=0.57,95 \% \mathrm{CI} 0.42$ to 0.77$)$, and $\mathrm{GH}(\mathrm{HR}=0.78,95 \% \mathrm{CI} 0.64$ to 0.95$)$. Conclusions. Low-dose NOACs were comparable to standard-dose NOACs considering risks of ischemic stroke, major bleeding, ICH, and GH, and they were superior to warfarin. Low-dose NOACs might be prescribed effectively and safely for patients with AF. Considering limitations, further well-designed prospective studies are foreseen.

\section{Introduction}

Atrial fibrillation (AF), known as a common cardiac arrhythmia worldwide, can cause ischemic stroke and seriously jeopardize the health of global elder patients [1]. For decades, warfarin was prescribed to prevent ischemic stroke from AF by decreasing the production of several clotting proteins that rely on vitamin $\mathrm{K}$ [2]. However, the adherence to warfarin is severely affected by the frequent international normalized ratio (INR) monitoring, drugdrug and drug-food interactions [3]. In recent years, the approval of non-vitamin $\mathrm{K}$ antagonist oral anticoagulants
(NOACs), which directly inhibit the critical factors of the coagulation cascade, provided new anticoagulant strategies for the patients with AF.

Meta-analyses of RCTs assessed the efficacy and safety of standard-dose NOACs, low-dose NOACs, and warfarin in patients with AF. Moreover, the results revealed that lowdose NOACs were inferior to standard-dose NOACs in the efficacy with a higher risk of ischemic stroke and had no superior efficacy and safety than warfarin $[4,5]$; standarddose NOACs were superior to warfarin in the efficacy and safety with less ischemic stroke, mortality, and ICH [4-6]. However, low-dose NOACs are still frequently prescribed 
for patients with AF. Low-dose NOACs were prescribed for $31 \%, 19 \%$, and $29 \%$ of patients in Korea [7], France [8], and America [9], respectively. RCTs were performed under optimized conditions, strict inclusion and exclusion criteria, which might not reflect real-world conditions. Moreover, RCTs enroll a small, nonrepresentative subset of patients and overlook the important interactions between the patients and the real world, which can affect treatment outcomes [10]. Furthermore, medication adherence, the key point for treatment effectiveness, is closely monitored in RCTs, which is not always the case in clinical practice [10]. Real-world cohort studies, which enroll patients of broad-spectrum baseline characteristics, may provide a comprehensive picture of the clinical practice. Therefore, we aimed to further investigate the efficacy and safety of low-dose NOACs by conducting a meta-analysis of all relevant cohort studies.

\section{Methods}

This meta-analysis was prepared according to the PRISMA (Preferred Reporting Items for Systemic Reviews and Metaanalysis) and MOOSE (Meta-Analysis of Observational Studies in Epidemiology) guidelines [11, 12].

\subsection{Search Strategy and Study Selection. Cochrane Central} Register of Controlled Trials (CENTRAL) (from inception to September 9, 2021), MEDLINE (from inception to September 9, 2021), and Embase (from inception to September $9,2021)$ were systematically searched. Details of the search strategy are illustrated in Supplementary Tables S1-S3.

We developed inclusion criteria for this meta-analysis prospectively. The criteria of studies screening were as follows: (1) the target population was patients with AF; (2) studies involved lose-dose NOACs and standard-dose NOACs or warfarin; (3) studies included efficacy (ischemic stroke and mortality) or safety outcomes (major bleeding, intracranial hemorrhage, and gastrointestinal hemorrhage); (4) the study type was the cohort. AF patients with valvular heart disease (VHD) or receiving NOACs after catheter ablation and studies published in the forms of conference abstracts, letters, or protocols were excluded. In addition, for the same data source or overlapping data reported in more than one study, only the most comprehensive data with the longest follow-up period was included. References of included studies and relevant meta-analyses were screened for additional eligible studies as well.

2.2. Definitions of Low-Dose NOACs, Standard-Dose NOACs, and Warfarin. Definitions were in accordance with the included studies. Standard-dose NOACs and warfarin were defined as dabigatran $150 \mathrm{mg}$ b.i.d., rivaroxaban $20 \mathrm{mg}$ q.d., apixaban $5 \mathrm{mg}$ b.i.d., and edoxaban $60 \mathrm{mg}$ q.d., and INR of 2.0-3.0 [13]. Low-dose NOACs were defined as dabigatran $110 / 75 \mathrm{mg}$ b.i.d., rivaroxaban $15 / 10 \mathrm{mg}$ q.d., apixaban $2.5 \mathrm{mg}$ b.i.d., and edoxaban $30 \mathrm{mg}$ q.d. $[4,9]$. And for patients with creatinine clearance rate $(\mathrm{CrCl})$ of $30-50 \mathrm{~mL} / \mathrm{min}$, age $\geq 70$ years, and a prior history of bleeding, standard-dose dabigatran was defined as $110 \mathrm{mg}$ b.i.d. [14, 15]; for patients with $\mathrm{CrCl}$ of $15-50 \mathrm{~mL} / \mathrm{min}$, standard-dose rivaroxaban was defined as $10 \mathrm{mg}$ q.d. [16, 17]; for patients with any two of the following characteristics, $\geq 80$ years old, bodyweight $<60 \mathrm{~kg}$, and serum creatinine level $(\mathrm{Cr}) \geq 1.5 \mathrm{mg} / \mathrm{dL}$, standard-dose apixaban was defined as $2.5 \mathrm{mg}$ b.i.d. [18, 19]; for patients with $\mathrm{CrCl}$ of $15-50 \mathrm{~mL} / \mathrm{min}$ or bodyweight $<60 \mathrm{~kg}$, standard-dose edoxaban was defined as $30 \mathrm{mg}$ q.d. [20].

2.3. Data Extraction and Quality Assessment. The primary efficacy outcome was ischemic stroke, and the secondary efficacy outcome was mortality (all-cause mortality). The primary safety outcome was major bleeding, defined as fatal bleeding or bleeding in a critical site, and the secondary safety outcomes were intracranial hemorrhage (ICH) and gastrointestinal hemorrhage $(\mathrm{GH})$.

Two reviewers (Ze Li and Xiaozhen Wang) independently screened titles and abstracts of the retrieved studies to exclude those that did not explore questions of interest and then independently screened full texts of the remaining studies to identify those that met all the inclusion criteria. We manually checked the reference list of each acquired article for relevant studies. For each included study, two reviewers independently extracted the characteristics of the included studies and patients, as well as outcomes as predefined. Discrepancies were resolved by discussing with the third reviewer (Aiping Wen).

Bias risks were assessed with the Newcastle-Ottawa quality assessment scale [21]. The publication bias was quantitatively assessed by the Begg's [22] and Egger's tests [23], $P<0.05$ was taken as statistically significant. Two reviewers ( $\mathrm{Ze} \mathrm{Li}$ and Xiaozhen Wang) assessed risks of bias independently and in duplicate. Any disagreements were resolved in consultation with the supervisor (Aiping Wen).

2.4. Data Synthesis and Statistical Analysis. Intention to treat analysis (ITT) results were used wherever possible. If ITT results were not available, we used the data the author reported. All analyses were performed by Stata 16.0. Hazard ratios (HRs) and corresponding 95\% confidence intervals (CIs) were estimated with the random-effect model. The heterogeneity among studies was assessed by $I^{2}$ with $<25 \%, 25-50 \%$, and $>50 \%$ indicating low, moderate, and high degree of heterogeneity, respectively. Meta-regression analyses were performed to examine possible sources of heterogeneity in the data.

To explore the influence for different regions of patients, subgroup meta-analyses were performed by stratifying patients into Asia and non-Asia. Most cohort studies used the propensity score matching (PSM) method or multivariable model (MM) to balance the confounding factors between groups and minimize the heterogeneity, so we enrolled adjusted cohort studies to perform adjusted subgroup metaanalyses. For all comparisons in this meta-analysis, $P<0.05$ was taken as statistically significant.

\section{Results}

3.1. Studies Identification and Characteristics. A total of 2846 publications were identified through database search 
(Figure 1). After the study screening process, twenty-five cohort studies meeting the inclusion criteria were included.

In general, there were 487856 patients in all enrolled studies. 238292 patients were included in the standard-dose group, including 115518 patients receiving NOACs and 122774 patients receiving warfarin, and 249564 patients were involved in the low-dose NOACs group. The baseline characteristics of included studies are shown in Table 1. The detailed previous medical history and group contents of included studies are illustrated in Supplementary Tables S4 and S5.

3.2. Risks of Bias Assessment. Results of bias assessments are summarized in Supplementary Tables S6 and S7. Overall, most cohort studies reported low risks of bias, while seven studies did not balance the confounding factors between groups, which had risks of comparability bias $[8,25,30,31,39-41]$. Three studies did not report the length of follow-up [27, 31, 35], and most studies did not show the lost follow-up rate, which had risks of outcome bias. In addition, there was no publication bias for this meta-analysis by the Begg's and Egger's tests, except for the risk of ICH $(P=0.035$, Egger's test $)$ in the comparison of low-dose NOACs versus warfarin.

\subsection{Low-Dose NOACs versus Standard-Dose NOACs.} There was no significant difference between low-dose NOACs and standard-dose NOACs for risks of ischemic stroke (HR $=1.03,95 \%$ CI 0.96 to $1.11, I^{2}=0 \%$ ), major bleeding ( $\mathrm{HR}=1.12,95 \% \mathrm{CI} 0.97$ to $\left.1.28, I^{2}=52.3 \%\right)$, ICH $\left(\mathrm{HR}=1.09,95 \%\right.$ CI 0.88 to $\left.1.36, I^{2}=33.2 \%\right)$, and $\mathrm{GH}$ $\left(\mathrm{HR}=1.11,95 \% \mathrm{CI} 0.92\right.$ to $\left.1.33, I^{2}=65.0 \%\right)$. However, compared with standard-dose NOACs, low-dose NOACs were associated with a higher risk of mortality $(\mathrm{HR}=1.41$, $95 \%$ CI 1.21 to $\left.1.65, I^{2}=78.2 \%\right)$. And results of Asia and non-Asia subgroup meta-analyses were also the same to the overall (Figure 2). Details of subgroup meta-analyses are illustrated in Supplementary Figures S1-S5.

To minimize the heterogeneity and obtain more reliable results, adjusted subgroup meta-analyses including cohort studies with the PSM or MM method were performed. Results of all outcomes were consistent with the overall meta-analysis as well. Details of adjusted subgroup metaanalyses are illustrated in Supplementary Figures S6-S9.

For meta-regression analyses, no significant correlations were observed in most efficacy and safety outcomes. However, a significant correlation was found between major bleeding and mean age $(P=0.010)$, with HR increasing as the mean age of included patients ascended (Supplementary Figure S10); other significant predictors of HR were found between ICH, mean age $(P=0.046)$, and female $(P=0.035)$ as well, with HR increasing as the mean age (Supplementary Figure S11) or female percent of included patients ascended (Supplementary Figure S12). Details of meta-regression analyses are illustrated in Supplementary Table S8.

To balance the confounding factors, subgroup metaanalyses stratified by mean age (divided into older and younger groups by median) were performed, respectively. In

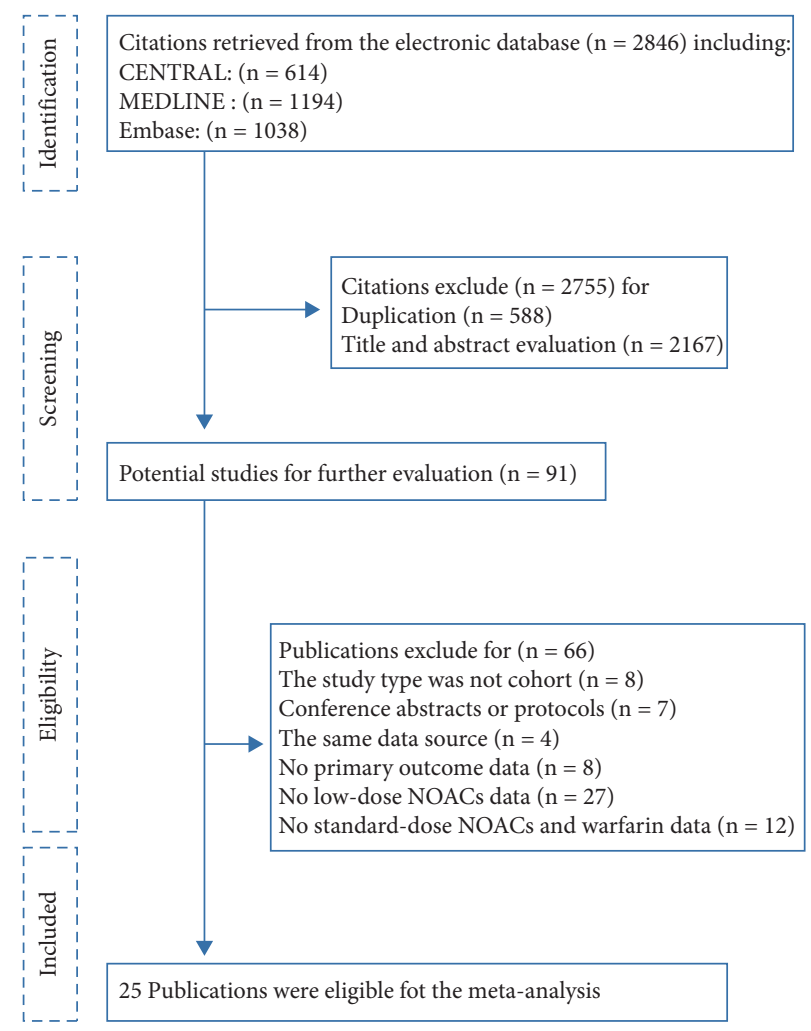

Figure 1: Flow-chart for the selection of included studies.

general, all results were consistent with the overall metaanalysis. Details of subgroup meta-analyses are shown in Supplementary Figures S13 and S14.

3.4. Low-Dose NOACs versus Warfarin. Compared with warfarin, low-dose NOACs were associated with lower risks of ischemic stroke (HR $=0.72,95 \% \mathrm{CI} .67$ to $0.78, I^{2}=2.1 \%$ ), mortality ( $\mathrm{HR}=0.67,95 \%$ CI 0.59 to $0.77, I^{2}=77.8 \%$ ), major bleeding ( $\mathrm{HR}=0.64,95 \% \mathrm{CI} 0.53$ to $0.79, I^{2}=71.8 \%$ ), ICH $\left(\mathrm{HR}=0.57,95 \%\right.$ CI 0.42 to $\left.0.77, I^{2}=69.5 \%\right)$, and $\mathrm{GH}$ ( $\mathrm{HR}=0.78,95 \%$ CI 0.64 to $\left.0.95, I^{2}=45.6 \%\right)$. And results of Asia and non-Asia subgroup meta-analyses were similar to the overall except for the comparable risk of $\mathrm{GH}(\mathrm{HR}=0.92$, 95\% CI 0.51 to $1.66, I^{2}=0 \%$ ) in non-Asia (Figure 3). Details of subgroup meta-analyses are shown in Supplementary Figures S15-S19.

Results of adjusted subgroup meta-analyses were consistent with the overall as well. Details of adjusted subgroup meta-analyses are illustrated in Supplementary Figures S20 and S21. For meta-regression analyses, no significant correlations were observed in efficacy and safety outcomes. Details of meta-regression analyses are illustrated in Supplementary Table S9.

\section{Discussion}

To the best of our knowledge, this is the first meta-analysis of cohort studies for low-dose NOACs versus standard-dose NOACs or warfarin in patients with AF. A few previous meta-analyses had tried to assess the efficacy and safety of 


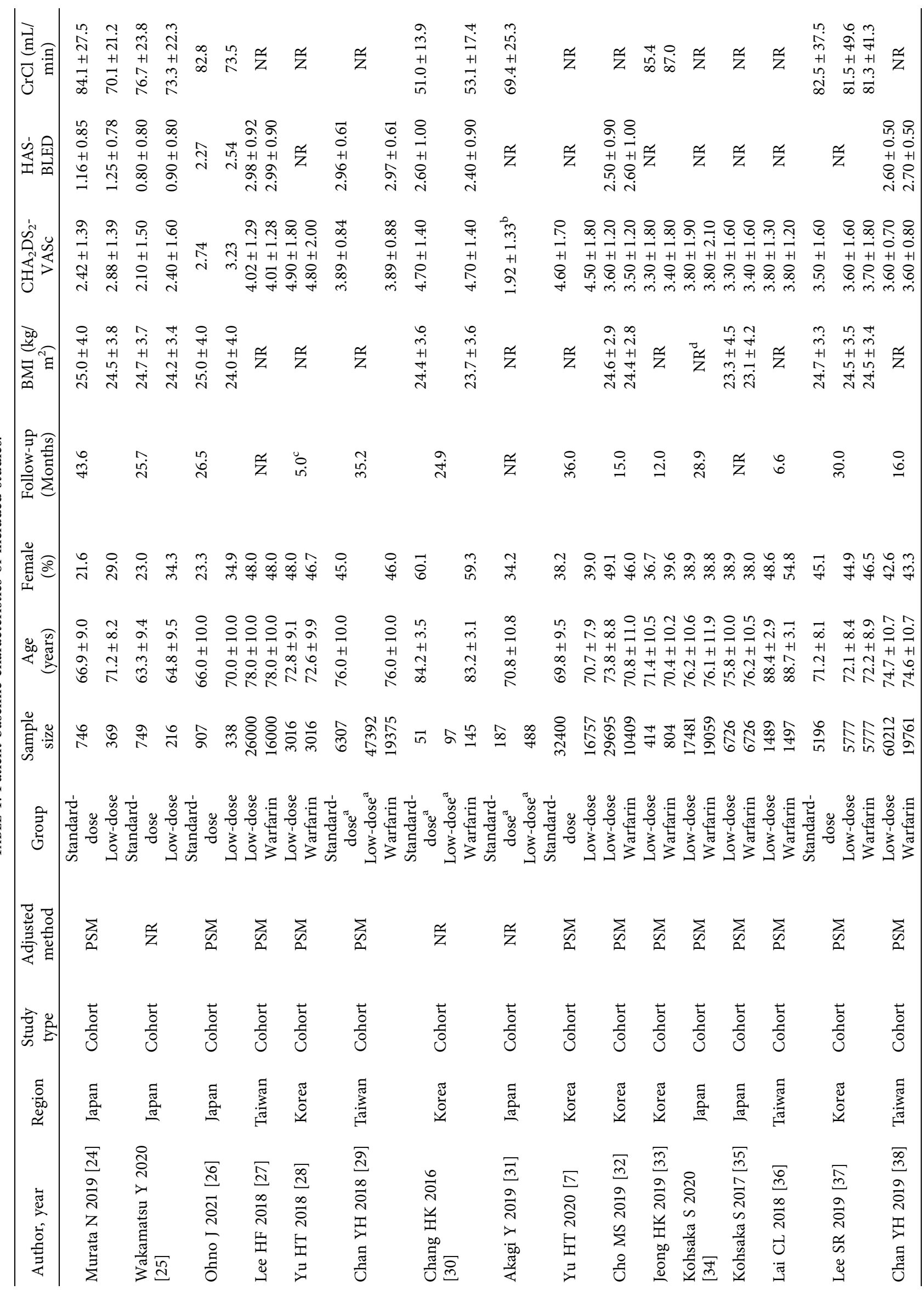




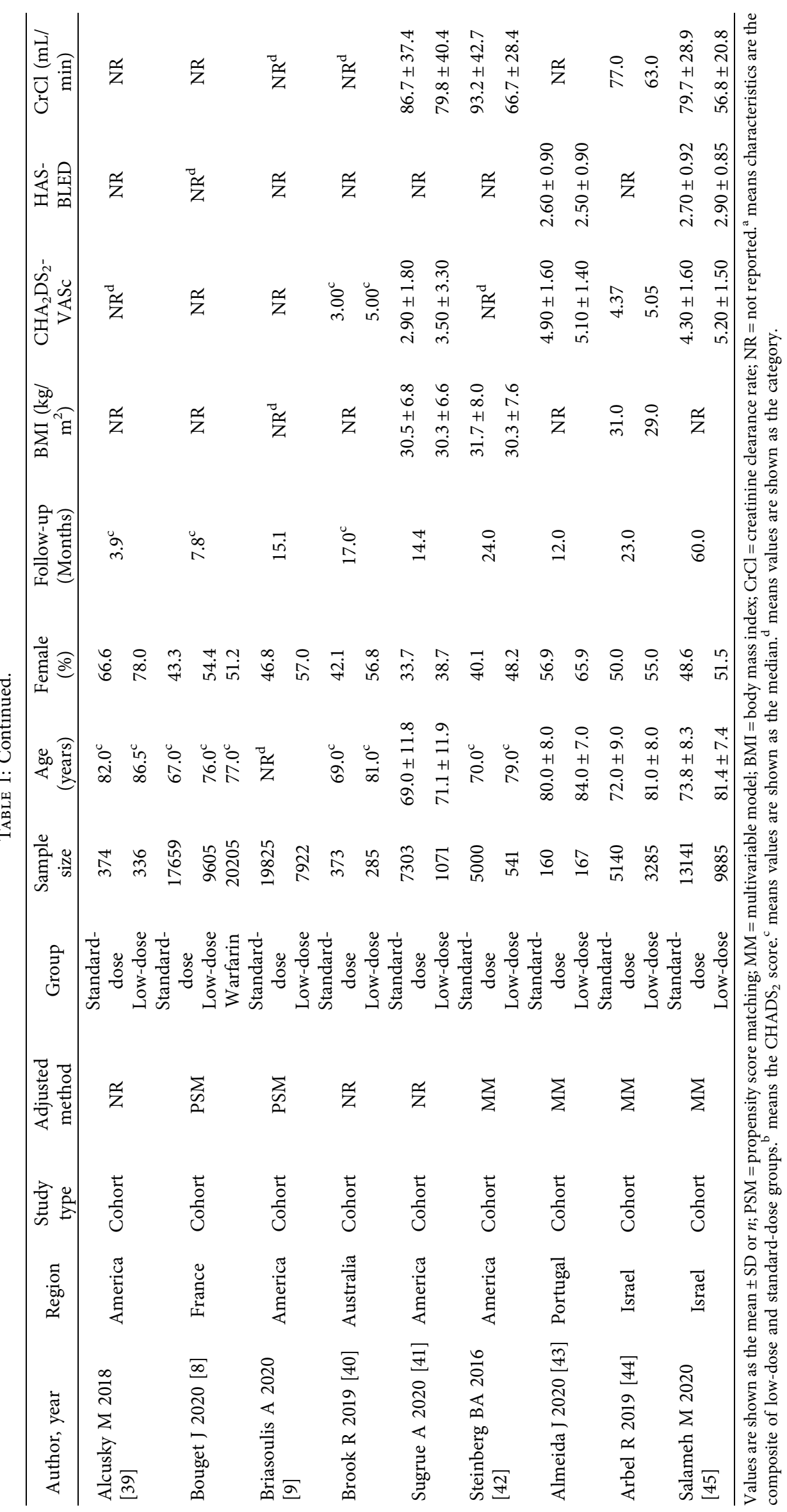




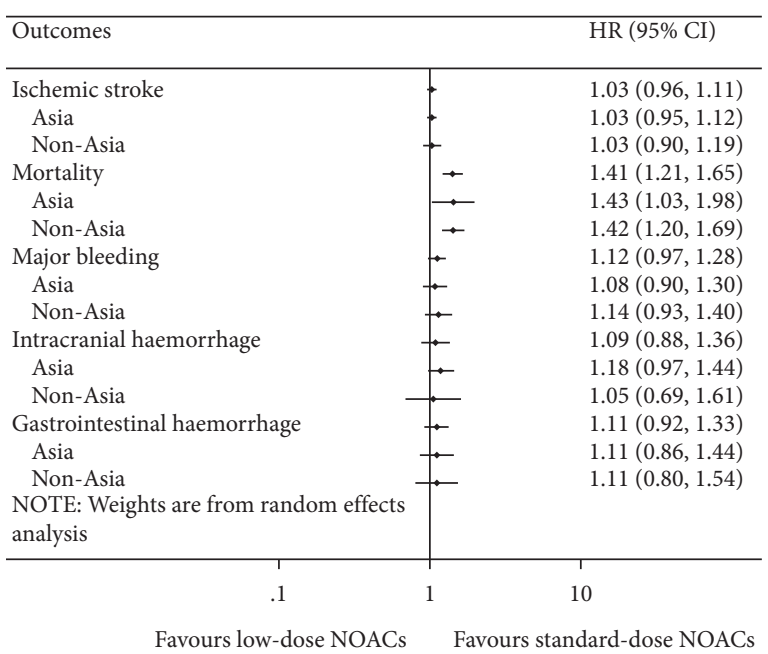

Figure 2: Meta-analyses of the efficacy and safety for low-dose NOACs versus standard-dose NOACs. HR=hazard ratio and $\mathrm{CI}=$ confidence interval.

\begin{tabular}{|c|c|c|}
\hline \multicolumn{2}{|l|}{ Outcomes } & \multirow{2}{*}{$\begin{array}{l}\mathrm{HR}(95 \% \mathrm{CI}) \\
0.72(0.67,0.78)\end{array}$} \\
\hline Ischemic stroke & + & \\
\hline Mortality & $\rightarrow$ & $0.67(0.59,0.77)$ \\
\hline Asia & $\rightarrow$ & $0.66(0.56,0.78)$ \\
\hline Non-Asia & $\rightarrow$ & $0.71(0.59,0.85)$ \\
\hline Major bleeding & $\rightarrow$ & $0.64(0.53,0.79)$ \\
\hline Asia & $\rightarrow$ & $0.66(0.53,0.81)$ \\
\hline Non-Asia & $\rightarrow$ & $0.49(0.26,0.94)$ \\
\hline Intracranial haemorrhage & $\rightarrow$ & $0.57(0.42,0.77)$ \\
\hline Asia & $\rightarrow$ & $0.58(0.42,0.81)$ \\
\hline Non-Asia & . & $0.44(0.20,0.99)$ \\
\hline Gastrointestinal haemorrhage & $\rightarrow$ & $0.78(0.64,0.95)$ \\
\hline Asia & $\rightarrow$ & $0.76(0.61,0.95)$ \\
\hline Non-Asia & $\longrightarrow$ & $0.92(0.51,1.66)$ \\
\hline \multicolumn{2}{|c|}{$\begin{array}{l}\text { NOTE: Weights are from random effects } \\
\text { analysis }\end{array}$} & \\
\hline .1 & 1 & 10 \\
\hline
\end{tabular}

FIGURE 3: Meta-analyses of the efficacy and safety for low-dose NOACs versus warfarin. $\mathrm{HR}=$ hazard ratio and $\mathrm{CI}=$ confidence interval.

low-dose NOACs by RCTs $[4,5]$. And the results indicated that when compared with standard-dose NOACs, low-dose NOACs showed the inferior efficacy with a higher risk of ischemic stroke; when compared with warfarin, low-dose NOACs showed the comparable efficacy and safety. Even though the meta-analysis of RCTs is the highest level of evidence, results of cohort studies may better represent the clinical practice with additional real-world data. For example, the previous meta-analyses of RCTs only enrolled patients of approximately 70 years old with the standard weight of roughly $66 \mathrm{~kg}$ [4-6]. These may not be generalizable to underrepresented patients, such as the patients with low weight, older age, or who were not yet represented in RCTs, so we performed this meta-analysis.

Our meta-analysis revealed that compared with standard-dose NOACs, low-dose NOACs had comparable risks of ischemic stroke and bleeding (including major bleeding, $\mathrm{ICH}$, and GH), except for a higher risk of mortality; compared with warfarin, low-dose NOACs showed lower risks of ischemic stroke, mortality, and bleeding. To assess the influence of different regions, we stratified the patients into the Asia subgroup and non-Asia subgroup. Results of subgroup meta-analyses were consistent with the overall except for the comparable risk of $\mathrm{GH}$ for the non-Asia subgroup in the comparison of low-dose NOACs versus warfarin.

We need to note that the baseline characteristics of cohort studies may be diverse compared to RCTs. Concerning some included studies, the mean or median ages of low-dose NOACs group were much older than standard-dose NOACs group, which led to the relatively lower $\mathrm{CrCL}$, higher $\mathrm{CHA}_{2} \mathrm{DS}_{2}$-VASc and HAS-BLED scores [8, 24, 26, 32, 40, 42-45]. Moreover, there were some heterogeneities in the previous medical history, including hypertension, diabetes, heart failure, vascular disease, stroke/transient ischemic attack (TIA), and major bleeding. Due to the broad-spectrum baseline characteristics, most cohort studies used the PSM or MM method to adjust the data and minimize the heterogeneity. Adjusted subgroup metaanalyses including cohort studies with PSM or MM were performed as well, and the results were consistent with the overall meta-analysis.

Meta-regression analyses indicated that the mean age and female percent of included patients captured a very substantial portion of the heterogeneity in the data, so subgroup meta-analyses stratified by those were performed to balance the confounding factors. Similarly, the results were consistent with the overall as well. Nonetheless, considering the relatively few studies and ineluctable heterogeneity in this meta-analysis, further high qualified prospective studies are required to validate these results.

Most of our results were similar to the previous metaanalyses of RCTs. However, there were some conflicting results in our meta-analysis compared with RCTs, such as the comparable risk of ischemic stroke and higher risk of mortality in the comparison of standard-dose NOACs, and lower risks of ischemic stroke, major bleeding, and $\mathrm{GH}$ in the comparison of warfarin $[4,5]$. The difference in outcomes could be partially explained by several reasons: firstly, the patients' baselines of RCTs were narrow and nonrepresentative, with the approximate age of 70 years old, bodyweight of $66 \mathrm{~kg}$, female percent of $40 \%$, and $\mathrm{CHADS}_{2}$ score of 2.0-3.0 $[4,5]$. These might only address a special population of AF patients. On the contrary, cohort studies in this meta-analysis presented broad-spectrum baseline characteristics, with age ranging from 63.3 to 88.7 years old, BMI ranging from 23.1 to 31.7, $\mathrm{CHA}_{2} \mathrm{DS}_{2}$-VASc and HAS-BLED scores ranging from 1.9 to $5.2,0.8$ to 3.0 , respectively. Compared with RCTs, cohort studies involved the individual of older age, lower body weight and $\mathrm{CrCL}$, or higher $\mathrm{CHA}_{2} \mathrm{DS}_{2}$-VASc and HAS-BLED scores, who might be more susceptible to low-dose NOACs. Secondly, the adherence to standard-dose NOAC was about $60 \%$, and more than one-third of patients with label NOAC prescription received a reduced low-dose [46]. As a result, some patients might be prescribed for standard-dose NOACs, whereas they take low-dose NOACs in reality. We believed that the above two reasons might contribute to the 
noninferiority of low-dose NOACs versus standard-dose NOACs in the real world. Thirdly, the mean or median ages of low-dose NOACs were much older than standard-dose NOACs in nine studies [8,24, 26, 32, 40,42-45], and we considered this might explain the higher risk of mortality for low-dose NOACs. As another study showed, the older patients with AF were faced more comorbidities and death factors [47], which might eventually result in the higher risk of mortality. In addition, as it was not convenient to monitor the quality of warfarin routine usage, and many patients cannot reach the baseline requirement of time in therapeutic range (TTR) [48]; this might lead to the superiority of low-dose NOACs versus warfarin in clinical practice.

Warfarin showed some therapeutic limitations in the clinical practice, whose effect was widely affected by food and drugs, and patients need to monitor the INR frequently to supervise the efficacy and risk of major bleeding [49]. Major bleeding can seriously affect the anticoagulation treatment, such as higher risks of stroke and mortality [50], longer hospitalization [51], and more health care resource utilization [52]. At the same time, patients taking warfarin often had less time within the therapeutic range [48]. In this meta-analysis, low-dose NOACs were noninferior to standard-dose NOACs and superior to warfarin. Thus, considering the excellence and convenience, low-dose NOACs could be an effective and safe alternative to warfarin.

4.1. Limitations. However, there were some potential limitations for our meta-analysis. Firstly, due to the limited number of included studies, we pooled all NOACs together even though rivaroxaban, apixaban, and edoxaban are the factor Xa inhibitors [53], while dabigatran is the thrombin inhibitor [54], which was conducted in another meta-analysis [55]. This may not cause significant bias, for they are all directacting oral anticoagulants inhibiting the critical factors in the coagulation cascade. Secondly, this meta-analysis might have some fundamental heterogeneity due to the nature of cohort studies, such as the mean age, $\mathrm{CHA}_{2} \mathrm{DS}_{2}$-VASc and HASBLED scores. However, most studies had used the PSM or MM method to adjust the data and minimize the heterogeneity. In addition, the results of adjusted subgroup metaanalyses including studies with PSM or MM were consistent with the overall as well. Thirdly, most included studies did not report the quality of TTR for warfarin. As the efficacy of warfarin was affected by the TTR, many patients cannot reach the baseline requirement of TTR [48], which might lead to the unexpected bias of low-dose NOACs versus warfarin. This limitation could be found in other meta-analyses involving warfarin $[55,56]$. However, the effectiveness of the treatment is ensured not only by effective and potent drugs, but also by patients' adherence to the therapy [57], and we should have various and comprehensive views of this limitation.

\section{Conclusions}

In general, for patients with $\mathrm{AF}$, this meta-analysis of cohort studies demonstrated that low-dose NOACs were comparable to standard-dose NOACs considering risks of ischemic stroke, major bleeding, ICH, and GH, and they were superior to warfarin. Thus, low-dose NOACs might be prescribed effectively and safely for patients with AF. However, considering limitations, further well-designed prospective studies are required to validate these results.

\section{Data Availability}

All data generated or analyzed during this study are included in this published article and its supplementary information files.

\section{Ethical Approval}

This is a meta-analysis and needs no ethical committee approval.

\section{Conflicts of Interest}

The authors declare that there are no conflicts of interest.

\section{Authors' Contributions}

Ze Li was responsible for the study design, literature search, data collection, data analysis, data interpretation, drafting, and critical revision of the manuscript. Xiaozhen Wang and Dandan Li were responsible for the literature search and data collection. Aiping Wen was responsible for the study concept and design, data interpretation, critical revision of the manuscript, approval of the final submission, and took responsibility for the data's integrity and accuracy.

\section{Acknowledgments}

This work was supported by the Digestive Medical Coordinated Development Center of Beijing Municipal Administration of Hospitals No. XXZ06.

\section{Supplementary Materials}

Supplementary Table S1. Cochrane Central Register of Controlled Trials search strategy. Supplementary Table S2. Embase search strategy. Supplementary Table S3. MEDLINE search strategy. Supplementary Table S4. Detailed previous medical history of included patients. Supplementary Table S5. Detailed group contents of included studies. Supplementary Table S6. Results of quality assessment using the NEWCASTLE-OTTAWA SCALE for cohort studies. Supplementary Table S7. Results of publication bias assessment for interesting outcomes. Supplementary Table S8. Results of meta-regression analyses for low-dose NOACs versus standard-dose NOACs. Supplementary Table S9. Results of meta-regression analyses for low-dose NOACs versus warfarin. Supplementary Figure S1. Pooled stroke of lowdose NOACs versus standard-dose NOACs. Supplementary Figure S2. Pooled mortality of low-dose NOACs versus standard-dose NOACs. Supplementary Figure S3. Pooled major bleeding of low-dose NOACs versus standard-dose NOACs. Supplementary Figure S4. Pooled ICH of low-dose NOACs versus standard-dose NOACs. Supplementary 
Figure S5. Pooled GH of low-dose NOACs versus standarddose NOACs. Supplementary Figure S6. Pooled adjusted stroke of low-dose NOACs versus standard-dose NOACs. Supplementary Figure S7. Pooled adjusted mortality of lowdose NOACs versus standard-dose NOACs. Supplementary Figure S8. Pooled adjusted major bleeding of low-dose NOACs versus standard-dose NOACs. Supplementary Figure S9. Pooled adjusted ICH of low-dose NOACs versus standard-dose NOACs. Supplementary Figure S10. Result of meta-regression between major bleeding and mean age for low-dose NOACs versus standard-dose NOACs. Supplementary Figure S11. Result of meta-regression between ICH and mean age for low-dose NOACs versus standard-dose NOACs. Supplementary Figure S12. Result of meta-regression between $\mathrm{ICH}$ and female for low-dose NOACs versus standard-dose NOACs. Supplementary Figure S13. Pooled major bleeding of low-dose NOACs versus standarddose NOACs stratified by mean age. Supplementary Figure S14. Pooled ICH of low-dose NOACs versus standard-dose NOACs stratified by mean age. Supplementary Figure S15. Pooled stroke of low-dose NOACs versus warfarin. Supplementary Figure S16. Pooled mortality of low-dose NOACs versus warfarin. Supplementary Figure S17. Pooled major bleeding of low-dose NOACs versus warfarin. Supplementary Figure S18. Pooled ICH of low-dose NOACs versus warfarin. Supplementary Figure S19. Pooled GH of low-dose NOACs versus warfarin. Supplementary Figure S20. Pooled adjusted stroke of low-dose NOACs versus warfarin. Supplementary Figure S21. Pooled adjusted major bleeding of low-dose NOACs versus warfarin. (Supplementary Materials)

\section{References}

[1] B. A. Williams, A. M. Chamberlain, J. C. Blankenship, E. M. Hylek, and S. Voyce, "Trends in atrial fibrillation incidence rates within an integrated health care delivery system, 2006 to 2018," JAMA Network Open, vol. 3, no. 8, Article ID e2014874, 2020.

[2] R. K. Wadhera, C. E. Russell, and G. Piazza, "Cardiology patient page. Warfarin versus novel oral anticoagulants: how to choose?” Circulation, vol. 130, no. 22, pp. e191-193, 2014.

[3] E. Bray, R. Georgiou, and N. Wilson, "Self-monitoring of INR for warfarin management of patients with atrial fibrillation (AF): patient and clinicians experiences," International Journal of Stroke, vol. 14, no. 4, p. 40, 2019.

[4] K. L. Wang, R. P. Giugliano, S. Goto et al., "Standard dose versus low dose non-vitamin $\mathrm{K}$ antagonist oral anticoagulants in Asian patients with atrial fibrillation: a meta-analysis of contemporary randomized controlled trials," Heart Rhythm, vol. 13, no. 12, pp. 2340-2347, 2016.

[5] B. Jia, H. S. Lynn, F. Rong, and W. Zhang, "Meta-analysis of efficacy and safety of the new anticoagulants versus warfarin in patients with atrial fibrillation," Journal of Cardiovascular Pharmacology, vol. 64, no. 4, pp. 368-374, 2014.

[6] C. T. Ruff, R. P. Giugliano, E. Braunwald et al., "Comparison of the efficacy and safety of new oral anticoagulants with warfarin in patients with atrial fibrillation: a meta-analysis of randomised trials," The Lancet, vol. 383, no. 9921, pp. 955-962, 2014.
[7] H. T. Yu, P. S. Yang, E. Jang et al., "Label adherence of direct oral anticoagulants dosing and clinical outcomes in patients with atrial fibrillation," Journal of American Heart Association, vol. 9, no. 12, Article ID e014177, 2020.

[8] J. Bouget, F. Balusson, M. Maignan et al., "Major bleeding risk associated with oral anticoagulant in real clinical practice. A multicentre 3-year period population-based prospective cohort study," British Journal of Clinical Pharmacology, vol. 86, no. 12, pp. 2519-2529, 2020.

[9] A. Briasoulis, Y. Gao, C. Inampudi et al., "Characteristics and outcomes in patients with atrial fibrillation receiving direct oral anticoagulants in off-label doses," BMC Cardiovascular Disorders, vol. 20, no. 1, p. 42, 2020.

[10] D. Price, G. Brusselle, N. Roche, D. Freeman, and A. Chisholm, "Real-world research and its importance in respiratory medicine," Breathe, vol. 11, no. 1, pp. 26-38, 2015.

[11] M. D. F. McInnes, D. Moher, B. D. Thombs et al., "Preferred reporting Items for a systematic review and meta-analysis of diagnostic test accuracy studies," JAMA, vol. 319, no. 4, pp. 388-396, 2018.

[12] D. F. Stroup, "Meta-analysis of observational studies in EpidemiologyA proposal for reporting," JAMA, vol. 283, no. 15, pp. 2008-2012, 2000.

[13] J. Steffel, P. Verhamme, T. S. Potpara et al., "The 2018 European Heart Rhythm Association Practical Guide on the use of non-vitamin $\mathrm{K}$ antagonist oral anticoagulants in patients with atrial fibrillation: executive summary," EP Europace, vol. 20, no. 8, pp. 1231-1242, 2018.

[14] S. J. Connolly, M. D. Ezekowitz, S. Yusuf et al., "Dabigatran versus warfarin in patients with atrial fibrillation," New England Journal of Medicine, vol. 361, no. 12, pp. 1139-1151, 2009.

[15] R. Govindarajan and E. Salgado, "Risk of bleeding with 2 doses of dabigatran compared with warfarin in older and younger patients with atrial fibrillation: an analysis of the randomized evaluation of long-term anticoagulant therapy (RE-LY) trial," Circulation, vol. 125, no. 3, pp. 293-294, 2012.

[16] M. R. Patel, K. W. Mahaffey, J. Garg et al., "Rivaroxaban versus warfarin in nonvalvular atrial fibrillation," New England Journal of Medicine, vol. 365, no. 10, pp. 883-891, 2011.

[17] M. Hori, M. Matsumoto, N. Tanahashi et al., "Rivaroxaban vs. Warfarin in Japanese patients with atrial fibrillation," Circulation Journal, vol. 76, no. 9, pp. 2104-2111, 2012.

[18] E. Szczerba, "Apixaban versus warfarin in patients with atrial fibrillation: examination of ARISTOTLE," Kardiologia Polska, vol. 70, no. 2, pp. 196-200, 2012.

[19] S. Ogawa, Y. Shinohara, and K. Kanmuri, "Safety and efficacy of the oral direct factor Xa inhibitor apixaban in Japanese patients with non-valvular atrial fibrillation," Circulation Journal, vol. 75, no. 8, pp. 1852-1859, 2011.

[20] J. Cunningham, R. Giugliano, E. Braunwald et al., "Edoxaban versus warfarin IN 841 patients with atrial fibrillation and peripheral arterial disease: insights from the engage af-timi 48 trial," Journal of the American College of Cardiology, vol. 67, no. 13, p. 2262, 2016.

[21] A. Stang, "Critical evaluation of the Newcastle-Ottawa scale for the assessment of the quality of nonrandomized studies in meta-analyses," European Journal of Epidemiology, vol. 25, no. 9, pp. 603-605, 2010.

[22] C. B. Begg and M. Mazumdar, "Operating characteristics of a rank correlation test for publication bias," Biometrics, vol. 50, no. 4, pp. 1088-1101, 1994.

[23] M. Egger, G. D. Smith, M. Schneider, and C. Minder, "Bias in meta-analysis detected by a simple, graphical test," $B M J$, vol. 315, no. 7109, pp. 629-634, 1997. 
[24] N. Murata, Y. Okumura, K. Yokoyama et al., "Clinical outcomes of off-label dosing of direct oral anticoagulant therapy among Japanese patients with atrial fibrillation identified from the SAKURA AF registry," Circulation Journal, vol. 83, no. 4, pp. 727-735, 2019.

[25] Y. Wakamatsu, K. Nagashima, R. Watanabe et al., "Clinical outcomes of off-label underdosing of direct oral anticoagulants after ablation for atrial fibrillation," International Heart Journal, vol. 61, no. 6, pp. 1165-1173, 2020.

[26] J. Ohno, Y. Sotomi, A. Hirata, Y. Sakata, A. Hirayama, and Y. Higuchi, "Dose of direct oral anticoagulants and adverse outcomes in Asia," The American Journal of Cardiology, vol. 139, pp. 50-56, 2021.

[27] H. F. Lee, Y. H. Chan, and H. T. Tu, "The effectiveness and safety of low-dose rivaroxaban in Asians with non-valvular atrial fibrillation," International Journal of Cardiology, vol. 261, pp. 78-83, 2018.

[28] H. T. Yu, P. S. Yang, and T. H. Kim, "Impact of renal function on outcomes with edoxaban in real-world patients with atrial fibrillation," Stroke, vol. 49, no. 10, pp. 2421-2429, 2018.

[29] Y. H. Chan, L. C. See, H. T. Tu et al., "Efficacy and safety of apixaban, dabigatran, rivaroxaban, and warfarin in asians with nonvalvular atrial fibrillation," Journal of American Heart Association, vol. 7, no. 8, Article ID e008150, 2018.

[30] H. K. Chang, M. Kim, and J. Kim, "Real-world comparison of non-vitamin $\mathrm{K}$ antagonist oral anticoagulants and warfarin in Asian octogenarian patients with atrial fibrillation," Journal of Geriatric Cardiology, vol. 13, no. 7, pp. 566-572, 2016.

[31] Y. Akagi, T. Chiba, S. Uekusa et al., "Retrospective cohort study of the efficacy and safety of dabigatran: real-life dabigatran use including very low-dose $75 \mathrm{mg}$ twice daily administration," Journal of Pharmaceutical Health Care and Sciences, vol. 5, pp. 1-7, 2019.

[32] M. S. Cho, J. E. Yun, J. J. Park et al., "Outcomes after use of standard- and low-dose non-vitamin $\mathrm{K}$ oral anticoagulants in asian patients with atrial fibrillation," Stroke, vol. 50, no. 1, pp. 110-118, 2019.

[33] H. K. Jeong, K. H. Lee, H. W. Park et al., "Real world comparison of rivaroxaban and warfarin in Korean patients with atrial fibrillation: propensity matching cohort analysis," Chonnam Medical Journal, vol. 55, no. 1, pp. 54-61, 2019.

[34] S. Kohsaka, J. Katada, K. Saito et al., "Safety and effectiveness of non-vitamin $\mathrm{K}$ oral anticoagulants versus warfarin in realworld patients with non-valvular atrial fibrillation: a retrospective analysis of contemporary Japanese administrative claims data," Open heart, vol. 7, no. 1, Article ID e001232, 2020.

[35] S. Kohsaka, T. Murata, N. Izumi, J. Katada, F. Wang, and Y. Terayama, "Bleeding risk of apixaban, dabigatran, and lowdose rivaroxaban compared with warfarin in Japanese patients with non-valvular atrial fibrillation: a propensity matched analysis of administrative claims data," Current Medical Research and Opinion, vol. 33, no. 11, pp. 1955-1963, 2017.

[36] C. L. Lai, H. M. Chen, M. T. Liao, and T. T. Lin, "Dabigatran, rivaroxaban, and warfarin in the oldest adults with atrial fibrillation in taiwan," Journal of the American Geriatrics Society, vol. 66, no. 8, pp. 1567-1574, 2018.

[37] S. R. Lee, E. K. Choi, K. D. Han, J. H. Jung, S. Oh, and G. Y. H. Lip, "Optimal rivaroxaban dose in asian patients with atrial fibrillation and normal or mildly impaired renal function," Stroke, vol. 50, no. 5, pp. 1140-1148, 2019.

[38] Y. H. Chan, H. F. Lee, L. C. See et al., "Effectiveness and safety of four direct oral anticoagulants in asian patients with nonvalvular atrial fibrillation," Chest, vol. 156, no. 3, pp. 529-543, 2019.

[39] M. Alcusky, A. L. Hume, M. Fisher et al., "Dabigatran versus rivaroxaban for secondary stroke prevention in patients with atrial fibrillation rehabilitated in skilled nursing facilities," Drugs \& Aging, vol. 35, no. 12, pp. 1089-1098, 2018.

[40] R. Brook, O. Aswapanyawongse, M. Tacey, T. Kitipornchai, P. Ho, and H. Y. Lim, "Real-world direct oral anticoagulant experience in atrial fibrillation: falls risk and low dose anticoagulation are predictive of both bleeding and stroke risk," Internal Medicine Journal, vol. 50, no. 11, pp. 1359-1366, 2020.

[41] A. Sugrue, D. Sanborn, M. Amin et al., "Inappropriate dosing of direct oral anticoagulants in patients with atrial fibrillation," The American Journal of Cardiology, vol. 144, pp. 52-59, 2021.

[42] B. A. Steinberg, P. Shrader, L. Thomas et al., "Off-label dosing of non-vitamin $\mathrm{K}$ antagonist oral anticoagulants and adverse outcomes," Journal of the American College of Cardiology, vol. 68, no. 24, pp. 2597-2604, 2016.

[43] J. P. H. C. L. de Almeida, A. S. Martinho, A. Girão et al., "Novel anticoagulants in an older and frail population with atrial fibrillation: the effect of inappropriate dosing on clinical outcomes," European Geriatric Medicine, vol. 11, no. 5, pp. 813-820, 2020.

[44] R. Arbel, R. Sergienko, A. Hammerman et al., "Effectiveness and safety of off-label dose-reduced direct oral anticoagulants in atrial fibrillation," The American Journal of Medicine, vol. 132, no. 7, pp. 847-855, 2019.

[45] M. Salameh, N. Gronich, N. Stein et al., "Stroke and bleeding risks in patients with atrial fibrillation treated with reduced apixaban dose: a real-life study," Clinical Pharmacology \& Therapeutics, vol. 108, no. 6, pp. 1265-1273, 2020.

[46] S. R. Lee, Y. S. Lee, J. S. Park et al., "Label adherence for nonvitamin $\mathrm{K}$ antagonist oral anticoagulants in a prospective cohort of asian patients with atrial fibrillation," Yonsei Medical Journal, vol. 60, no. 3, pp. 277-284, 2019.

[47] S. Wu, Y. M. Yang, J. Zhu et al., "Impact of age on the association between body mass index and all-cause mortality in patients with atrial fibrillation," The Journal of Nutrition, Health \& Aging, vol. 21, no. 10, pp. 1125-1132, 2017.

[48] C. L. Morgan, P. Mcewan, A. Tukiendorf, P. A. Robinson, A. Clemens, and J. M. Plumb, "Warfarin treatment in patients with atrial fibrillation: observing outcomes associated with varying levels of INR control," Thrombosis Research, vol. 124, no. 1, pp. 37-41, 2009.

[49] G. DeSantis, J. Hogan-Schlientz, G. Liska et al., "STABLE results: warfarin home monitoring achieves excellent INR control," American Journal of Managed Care, vol. 20, no. 3, pp. 202-209, 2014.

[50] S. Deitelzweig, A. Keshishian, A. Kang et al., "Burden of major gastrointestinal bleeding among oral anticoagulant-treated non-valvular atrial fibrillation patients," Therapeutic advances in gastroenterology, vol. 14, pp. 1-13, 2021.

[51] N. S. Abraham and D. L. Castillo, "Novel anticoagulants," Current Opinion in Gastroenterology, vol. 29, no. 6, pp. 676-683, 2013.

[52] C. Sam, J. M. Massaro, R. B. D’Agostino et al., "Warfarin and aspirin use and the predictors of major bleeding complications in atrial fibrillation (The Framingham Heart Study)," The American Journal of Cardiology, vol. 94, no. 7, pp. 947951, 2004.

[53] P. Mismetti and S. Laporte, "Rivaroxaban: données pharmaco-cliniques," Annales Françaises d'Anesthesie et de Reanimation, vol. 27, no. 1, pp. S16-S21, 2008. 
[54] S. Spinler, "The pharmacology and therapeutic use of dabigatran etexilate," The Journal of Clinical Pharmacology, vol. 53, no. 1, pp. 1-13, 2013.

[55] F. Dentali, N. Riva, M. Crowther, A. G. G. Turpie, G. Y. H. Lip, and W. Ageno, "Efficacy and safety of the novel oral anticoagulants in atrial fibrillation," Circulation, vol. 126, no. 20, pp. 2381-2391, 2012.

[56] F. Rong, B. Jia, P. Huang, H. S. Lynn, and W. Zhang, "Safety of the direct-acting anticoagulants in patients with atrial fibrillation: a meta-analysis," Thrombosis Research, vol. 135, no. 6, pp. 1117-1123, 2015.

[57] C. Dagli-Hernandez, R. C. Lucchetta, T. R. de Nadai, J. C. Fernandez Galduróz, and P. de Carvalho Mastroianni, "Self-perception of knowledge and adherence reflecting the effectiveness of antiretroviral therapy," Patient Preference and Adherence, vol. 10, pp. 1787-1793, 2016. 